\title{
Capecitabine-Associated Terminal Ileitis
}

\author{
Irene E.G. van Hellemond Annemarie M. Thijs Geert-Jan Creemers \\ Department of Internal Medicine, Catharina Hospital, Eindhoven, The Netherlands
}

\section{Keywords}

Colorectal cancer · Capecitabine · Fluoropyrimidine - Terminal lleitis · Diarrhea · Adverse event

\begin{abstract}
Capecitabine is an oral fluoropyrimidine used as adjuvant and palliative chemotherapy in patients with colorectal cancer. Diarrhea is a well-known side effect of capecitabine and 5-fluorouracil agents. We present a case with terminal ileitis as a rare adverse event of capecitabine treatment. Capecitabine-induced terminal ileitis is likely to be underreported. It should be considered more often as a cause of severe and atypical complaints of diarrhea during treatment with capecitabine or other 5 -fluorouracil agents.

(C) 2018 The Author(s)

Published by S. Karger AG, Basel
\end{abstract}

\section{Introduction}

Capecitabine is an oral fluoropyrimidine, which is the chemotherapy backbone in adjuvant and palliative systemic treatment in patients with colorectal cancer. Gastrointestinal adverse events are common during capecitabine treatment, mostly consisting of nausea and diarrhea. In this paper, we present a rare case of capecitabine-associated terminal ileitis. 


\section{Case Reports in Oncology}

\section{Case Report}

A 69-year-old woman without any comorbidities underwent a sigmoid resection for an adenocarcinoma of the sigmoid colon (pT3N2). According to the Dutch guidelines, adjuvant chemotherapy was started 5 weeks after surgery with CAPOX, consisting of capecitabine and oxaliplatin every 3 weeks. Before start, dihydropyrimidine dehydrogenase genotype testing was performed without signs of DPD deficiency.

Shortly after the start of chemotherapy, she had complaints of nausea and a reduced appetite. After 2 weeks, she developed watery stools (without blood) twice daily. The diarrhea worsened to 4-6 times daily during the following days at which loperamide was administered. Three weeks after the start of chemotherapy, she was hospitalized. Physical examination revealed a moderately ill patient with normal vital functions. No abnormalities were found on examination of the heart and the lungs. Upon examination of the abdomen, lively bowel sounds were found, and the abdomen was distended but not tender at palpation. Digital rectal examination revealed no abnormalities. Also, examination of the skin and extremities showed no abnormalities.

The laboratory results showed a hemoglobin level of $8.3 \mathrm{mmol} / \mathrm{L}$ (reference 7.5-10.0), thrombocytes $300 / \mathrm{nL}$ (reference 150-400), leukocytes 6.0/nL (reference 4.0-10.0), and a Creactive protein of $83 \mathrm{mg} / \mathrm{L}$ (reference $<6$ ), with normal liver biochemistry and kidney function. The following days, the diarrhea worsened to a frequency of 12 times a day, and intravenous fluids were administered. Stool cultures were negative for pathogenic bacteria and protozoa. A colonoscopy with biopsy showed an ileitis with superficial but extensive ulceration in the terminal ileum (Fig. 1). Magnetic resonance enterography showed a 5-mm wall thickening of the terminal ileum over a distance of more than $15 \mathrm{~cm}$. More proximal in the ileum, a second area of wall thickness was found over a distance of $7 \mathrm{~cm}$. We discontinued the CAPOX treatment and started budesonide $9 \mathrm{mg}$ once daily. The diarrhea and nausea decreased in the following weeks. After 4 weeks of budesonide use, adjuvant treatment was continued with FOLFOX (leucovorin, 5-fluorouracil, and oxaliplatin) every 2 weeks. The complaints of nausea and diarrhea resumed mildly (grade 1) during FOLFOX therapy, and therefore we continued budesonide. After 3 cycles of FOLFOX, she decided to discontinue the adjuvant treatment because she was suffering from a vital depression. The budesonide was discontinued after cessation of the chemotherapy without signs of recurrence of ileitis.

\section{Discussion}

Diarrhea is a well-known, dose-dependent side effect of the treatment with capecitabine. The diarrhea usually starts at the end of the second or in the third week, is mostly mild, recovers after a few days with sometimes a necessity of treatment with anti-motility agents such as loperamide. Grade 3-4 diarrhea occurs in $11.4 \%$ of patients treated with capecitabine monotherapy [1]. In contrast, in patients with a DPD deficiency the diarrhea starts often within the first week and is life threatening [2].

Oxaliplatin can also cause diarrhea but typically starts early after administration within 3-4 days and resolves after 1-2 days without any intervention. In patients treated with the 
combination of capecitabine and oxaliplatin, grade 3-4 diarrhea occurs in approximately $18 \%$ of cases [1,3].

Capecitabine-induced diarrhea is caused by acute injury to the intestinal mucosa, which leads to loss of the epithelium [4]. By inducing a mitotic arrest of the crypt cells, the ratio of immature secretory crypt cells to mature villous enterocytes increases, leading to a higher volume of fluid leaving the small bowel that goes beyond the absorptive capacity of the colon, causing clinically significant diarrhea $[4,5]$.

In the current case, the complaints of diarrhea started within 2 weeks after the first administration of chemotherapy and worsened over time, needing further diagnostic tests, which revealed a terminal ileitis. Terminal ileitis has been previously reported in 8 cases (Table 1) [6-11]. Yet, the pathophysiology and the management of capecitabine-induced terminal ileitis remain unclear. Of the 9 reported cases, all received intravenous fluids, 2 received parenteral nutrition and 7 received antibiotics. Our patient was the only one treated with steroids. Because steroids seem to be contra-indicated in case of mucositis, we believe steroids should be given only after performing colonoscopy with biopsy revealing a clear diagnosis of ileitis.

Considering the cancer treatment, in 5 cases capecitabine was permanently discontinued; in 2 of these cases, a different type of chemotherapy was started, and in 3 cases no chemotherapy was resumed at all. In none of the cases was capecitabine re-administered the same dose; in 2 cases, capecitabine was restarted at a reduced dose, and in 1 case capecitabine was replaced by 5 -fluorouracil (FOLFOX).

If continuation of treatment is desired, patient and tumor characteristics should be taken into account. In a palliative setting, toxicity is an important factor influencing quality of life. Therefore, in case of capecitabine-induced terminal ileitis, resuming capecitabine in a palliative setting seems to be an inconsistent decision. In an adjuvant setting, the absolute risk reduction of relapse has to be weighted against the risk of toxicity. Hence, a re-challenge with a dose reduction of capecitabine or other 5 -fluorouracilagents might be a valid option.

An alternative treatment option could be S1, which is an oral fluoropyrimidine only registered for use in a palliative setting that includes three different agents: tegafur, gimeracil, and oteracil. S1 is associated with a significantly lower incidence of hand-foot syndrome compared with capecitabine, with comparable efficacy [12].

Another oral cytotoxic agent named trifluridine-tipiracil (TAS-102) is an oral fluoropyrimidine with a different mechanism of action, which is registered for patients with metastatic colorectal cancer who are refractory or intolerant to standard chemotherapy $[13,14]$. Trifluridine is a thymidine-based nucleic acid analogue; the triphosphate form of trifluridine interferes with DNA synthesis and inhibits cell proliferation. Tipiracil is a potent thymidine phosphorylase inhibitor, which prevents the rapid degradation of trifluridine, resulting in an increased trifluridine exposure [14]. Its associated toxicities are gastrointestinal and hematologic, but in contrast to capecitabine, the gastrointestinal toxicities with trifluridine-tipiracil were almost all grade 1 and 2 with only few grade $\geq 3$ events $[13,14]$. 


\section{Conclusion}

Terminal ileitis should be considered more often when the pattern of diarrhea and other complaints are not typical for capecitabine-induced mucositis. Continuing chemotherapy treatment with alternative fluoropyrimidines is an option.

\section{Statement of Ethics}

The authors have no ethical conflicts to disclose.

\section{Disclosure Statement}

The authors have nothing to disclose.

\section{References}

1 Iacovelli R, Pietrantonio F, Palazzo A, Maggi C, Ricchini F, de Braud F, et al. Incidence and relative risk of grade 3 and 4 diarrhoea in patients treated with capecitabine or 5 -fluorouracil: a meta-analysis of published trials. Br J Clin Pharmacol. 2014 Dec;78(6):1228-37.

2 Ezzeldin H, Diasio R. Dihydropyrimidine dehydrogenase deficiency, a pharmacogenetic syndrome associated with potentially life-threatening toxicity following 5 -fluorouracil administration. Clin Colorectal Cancer. 2004 Sep;4(3):181-9.

3 Baird R, Biondo A, Chhaya V, McLachlan J, Karpathakis A, Rahman S, et al. Toxicity associated with capecitabine plus oxaliplatin in colorectal cancer before and after an institutional policy of capecitabine dose reduction. Br J Cancer. 2011 Jan;104(1):43-50.

4 Ikuno N, Soda H, Watanabe M, Oka M. Irinotecan (CPT-11) and characteristic mucosal changes in the mouse ileum and cecum. J Natl Cancer Inst. 1995 Dec;87(24):1876-83.

5 Benson AB 3rd, Ajani JA, Catalano RB, Engelking C, Kornblau SM, Martenson JA Jr, et al. Recommended guidelines for the treatment of cancer treatment-induced diarrhea. J Clin Oncol. 2004 Jul;22(14):2918-26.

6 Radwan R, Namelo WC, Robinson M, Brewster AE, Williams GL. Ileitis secondary to oral capecitabine treatment? Case Rep Med. 2012;2012:154981.

7 Barton D. Ulcerative ileitis secondary to adjuvant capecitabine for colon cancer: a case report: UICC World Cancer Congress, Washington, July 2006, 84-41.

8 Bouma G, Imholz AL. [Ileitis following capecitabine use]. Ned Tijdschr Geneeskd. 2011;155:A3064.

9 Al-Gahmi AM, Kerr IG, Zekri JM, Zagnoon AA. Capecitabine-induced terminal ileitis. Ann Saudi Med. 2012 Nov-Dec;32(6):661-2.

10 Mokrim M, Aftimos PG, Errihani H, Piccart-Gebhart M: Breast cancer, DPYD mutations and capecitabinerelated ileitis: description of two cases and a review of the literature. BMJ Case Rep. 2014 Apr 19;2014. pii: bcr2014203647.

11 Lee SF, Chiang CL, Lee AS, Wong FC, Tung SY. Severe ileitis associated with capecitabine: two case reports and review of the literature. Mol Clin Oncol. 2015 Nov;3(6):1398-400.

12 Kwakman JJ, Simkens LH, van Rooijen JM, van de Wouw AJ, Ten Tije AJ, Creemers GJ, et al. Randomized phase III trial of S-1 versus capecitabine in the first-line treatment of metastatic colorectal cancer: SALTO study by the Dutch Colorectal Cancer Group. Ann Oncol. 2017 Jun;28(6):1288-93.

13 Yoshino T, Mizunuma N, Yamazaki K, Nishina T, Komatsu Y, Baba H, et al. TAS-102 monotherapy for pretreated metastatic colorectal cancer: a double-blind, randomised, placebo-controlled phase 2 trial. Lancet Oncol. 2012 Oct;13(10):993-1001. 


\section{Case Reports in Oncology}

Case Rep Oncol 2018;11:654-659

DOI: $10.1159 / 000492781$

(c) 2018 The Author(s). Published by S. Karger AG, Basel www.karger.com/cro

van Hellemond et al.: Capecitabine-Associated Terminal Ileitis

14 Mayer RJ, Van Cutsem E, Falcone A, Yoshino T, Garcia-Carbonero R, Mizunuma N, et al.; RECOURSE Study Group. Randomized trial of TAS-102 for refractory metastatic colorectal cancer. N Engl J Med. 2015 May;372(20):1909-19.
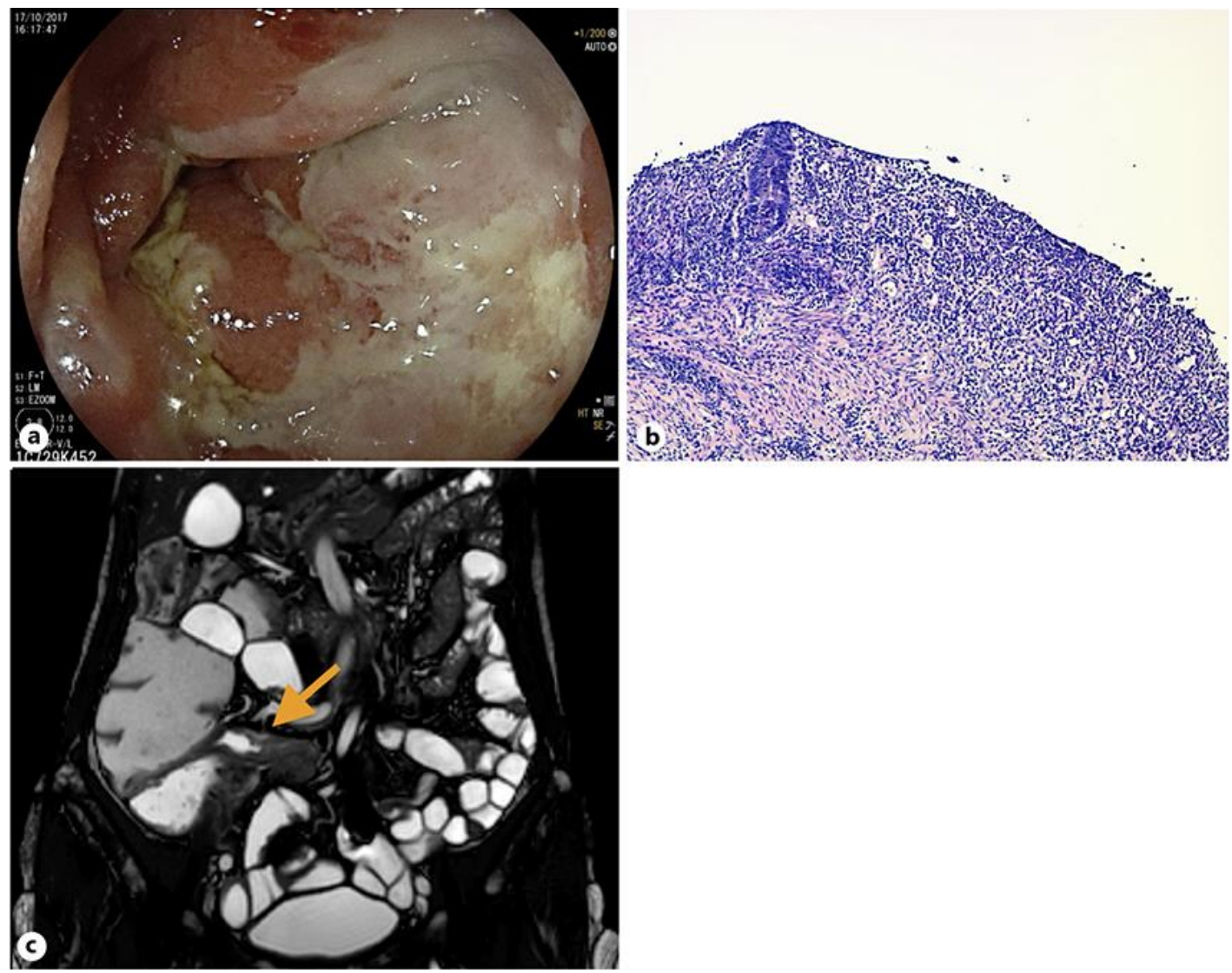

Fig. 1. Diagnostic findings. a Terminal ileitis at colonoscopy. b Extensive inflammation of the small intestine at pathological review of the biopsy. c MR enterography showing a distention of the colon and thickening of the terminal ileal loop (arrow). 
Table 1. Earlier reports on capecitabine associated terminal ileitis

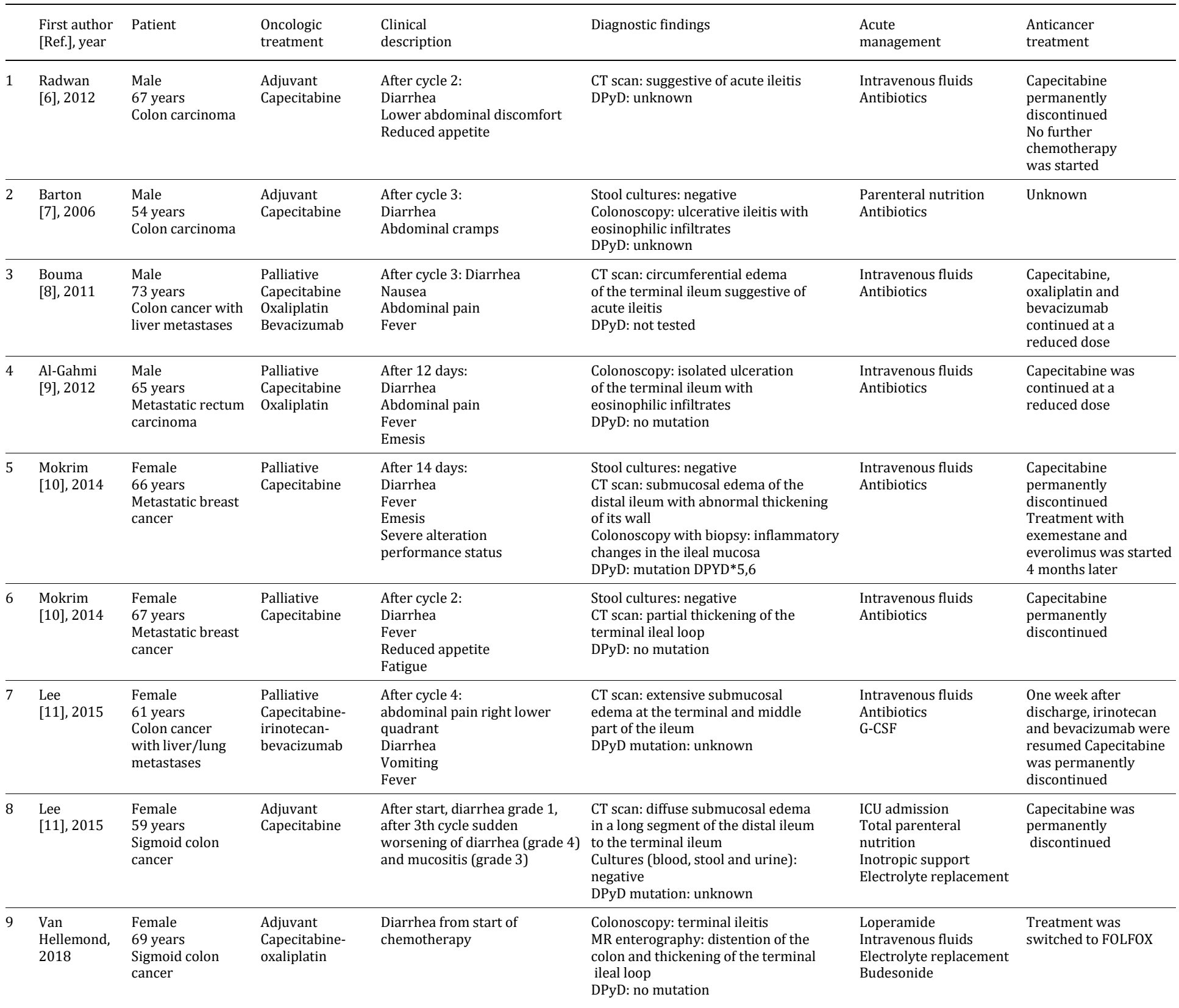

CT, computed tomography; DPyD, dihydropyrimidine dehydrogenase; FOLFOX, leucovorin, 5-fluorouracil, and oxaliplatin. 